\title{
CD5 expression by human ejaculated spermatozoa
}

\author{
Nasrin Sereshki ${ }^{1,2}$, Mitra Rafiee ${ }^{3}$, Razieh Alipour²*, David Wilkinson ${ }^{4}$ and Vahid Ahmadipanah ${ }^{1}$
}

\begin{abstract}
Background: A complex of effector and predominant regulatory immune responses are induced in the female reproductive tract (FRT) due to insemination that is necessary to achieve pregnancy. The expression of immune regulatory molecules by spermatozoa indicates the significance of the interaction between spermatozoa and immune cells recruited to the FRT in the preparation of appropriate immunity for pregnancy occurrence. One of the immune regulatory molecules is CD5 whose expression by spermatozoa has not yet been investigated. Therefore, the aim of this study is to investigate the expression of CD5 on the surface of human spermatozoa. Semen samples were collected from 30 healthy men with normal semen status. CD5 expression on purified spermatozoa was evaluated by flow cytometry methods.
\end{abstract}

Results: The results showed the mean \pm SD percentage of CD5 positive spermatozoa was $49.41 \pm 8.73$.

Conclusion: CD5 is expressed on spermatozoa.

Keywords: Immune regulatory factor, Spermatozoa, CD5

\section{Background}

A complex of effector and predominant regulatory immune responses are induced in the female reproductive tract (FRT) due to insemination that is necessary to achieve pregnancy [1-3]. Semen is composed of seminal fluid, spermatozoa and non-spermatozoa cells which include round cells and immune cells [4]. Seminal fluid contains immune regulatory factors such as prostaglandins, transforming growth factor $\beta$ (TGF- $\beta$ ), IL-10, and others [5]. In addition to seminal fluid immune regulatory factors, spermatozoa also express immune regulatory molecules. Human leukocyte antigen (HLA)-G [6], HLA-E [7], and Fas-ligand [8] are some examples of immunoregulatory molecules expressed by spermatozoa. The expression of such immune markers by spermatozoa indicates the significance of the interaction between spermatozoa and immune cells recruited to the FRT in the

\footnotetext{
*Correspondence: alipour55Razieh@gmail.com

${ }^{2}$ Department of Immunology, School of Medicine, Isfahan University

of Medical Sciences, Isfahan, Iran

Full list of author information is available at the end of the article
}

arrangement of proper immunity for pregnancy occurrence. It also raises the possibility of the presence of other immune molecules on spermatozoa. There is a variety of immune regulatory molecules such as cytotoxic $\mathrm{T}$ lymphocyte-associated protein 4 (CTLA-4), programmed cell death protein 1(PD1) and its ligand (PD-L1), $T$ cell immunoglobulin mucin-3 (tim3) [9], and CD5 [10] whose expression by spermatozoa, to the best of our knowledge, has not yet been investigated.

Given the great importance of the regulatory immune response to spermatozoa for a successful pregnancy and the obvious relationship between the immune dysregulation in the FRT and pregnancy disorders [11], elucidation of the immune molecules expressed by spermatozoa is necessary. Expanding our knowledge about the molecules involved in reproductive immunity can empower us to propose therapeutic interventions in immune dysregulation.

Among the above mentioned molecules, CD5 has particular importance because of its specific impact on the survival and receptor signaling of $\mathrm{T}$ and $\mathrm{B}$ lymphocytes, being the central cells in immune reactions [12]. CD5 is 
a scavenger receptor that is expressed by lymphocytes, primarily thymocytes, mature $\mathrm{T}$ cells, a subset of $\mathrm{B}$ cells (in mice B1a cells), and also by dendritic cells which are interacting with lymphocytes [13]. CD5 has a functional role in immune tolerance, modulating $\mathrm{T}$ helper differentiation as well as Tregs and regulatory B cells (Breg) homeostasis [13]. For example, a high expression of CD5 on T cells results in the induction of Treg cells. In contrast, lack of CD5 on T cells is accompanied with elevated activation and increased "activation-induced cell death" and anergy [14]. Development and function of B1a cells is dependent on CD5 expression [13]. CD5-positive dendritic cells lead to decreased activation of CD4- and CD8-positive $\mathrm{T}$ cells [14]. Accordingly, we supposed that CD5 may be one of the immune molecules which is expressed by spermatozoa to regulate $\mathrm{T}$ and $\mathrm{B}$ cell penetration to the FRT following insemination. Therefore, the aim of this study is to investigate the expression of CD5 on the surface of human spermatozoa.

\section{Methods \\ Subjects}

Thirty healthy volunteers aged 20-56years entered the study. Semen samples were collected by masturbation after 2-3 days of sexual abstinence. After semen analysis according to the WHO standard guidelines (WHO, 2010), samples with normal quality (according to WHO reference intervals for values of semen parameters) were selected for the assessment of CD5 expression.

\section{Sperm purification}

Density-gradient centrifugation technique was used for purification of spermatozoa. We used All Grad (Life Global ${ }^{\circledR}$ Group, Canada) solution. All Grad effectively separates the spermatozoa from debris, seminal plasma, epithelial cells, leukocyte, bacteria, and immature and abnormal spermatozoa [15]. The procedure of purification is described in more detail elsewhere [15]. In brief, $1 \mathrm{ml}$ of the spermatozoa suspension was carefully layered over a discontinuous gradient made by All Grad $95 \%$ and $45 \%$. After centrifugation at $400 \mathrm{~g}$ for $18 \mathrm{~min}$, the spermatozoa pellet at the bottom of the centrifuge tubes was washed and re-suspended in All Grad Wash. The purified spermatozoa were assessed by microscopic visualization for lack of non-spermatozoa cell contamination and also viability.

\section{Flow cytometry}

The presence of CD5 on the surface of spermatozoa was measured by direct immunofluorescence using a BD FACS Calibur (BD Biosciences, USA) flow cytometer. Tubes containing $1 \times 10^{6}$ spermatozoa were stained with phycoerythrin (PE) mouse anti-human CD5 (clone:
MCD5, IQ Products, Groningen, Netherlands) at room temperature for $30 \mathrm{~min}$. The samples were run on flow cytometry, and data from at least 100,000 events were collected using Cell Quest software (Becton Dickinson). Antibody titration was performed, and the optimal titer with the minimal background was selected. Unstained control was used as a negative control. Noteworthy, cell viability tests were not performed because abnormal and dead spermatozoa were removed by All Grad solution before staining. Fluorescence data were obtained with the logarithmic amplifier. We used a Flow Jo software version $\mathrm{X}$ for the data analysis.

\section{Results}

Flow cytometric assay was used for evaluating CD5 expression by spermatozoa. Due to the size of human spermatozoa, which are smaller than many other human cells, an appropriate semi-logarithmic setting was applied on the flow cytometer. In the setting, a logarithmic mode of the side scatter (SSC) parameter versus a liner mode of the forward scatter (FSC) parameter was used to detect spermatozoa, and a suitable gate was set around them. The corresponding histogram was used to determine the expression of CD5. The results clearly show the expression of CD5 on the surface of purified spermatozoa (Fig. 1). The mean \pm SD percentage of CD5 positive spermatozoa was $49.41 \pm 8.73$.

\section{Discussion}

The expression of CD5, an immune-associated molecule with immunomodulatory properties, has been demonstrated by some immune cells $[12,13]$. The finding of this study is noteworthy because, for the first time, it has been shown by the flow cytometry method that human spermatozoa display CD5 on their surface. A simple search in the Human Protein Atlas (https://www.proteinatlas.org/ ENSG00000110448-CD5/tissue) reveals CD5 expression in different tissues, including male and female reproductive tissues. However, the type of cells in the tissues which express CD5 molecules remains to be determined. Although the expression of CD5 by resident immune cells in the tissues can underlie the positivity for CD5, the results of the current study propose spermatozoa, in male reproductive tracts, as another possible CD5-expressing cell.

We do not know whether the flow cytometric detection of CD5 on the spermatozoa cell surface is due to CD5 mRNA translation or alternatively results from the acquisition of CD5 from other nearby cells through a mechanism called trogocytosis (already shown regarding CD9 acquisition of spermatozoa [16]). Further studies are needed to determine this. However, the presence of CD5 on spermatozoa, whether by the gene expression or by a 

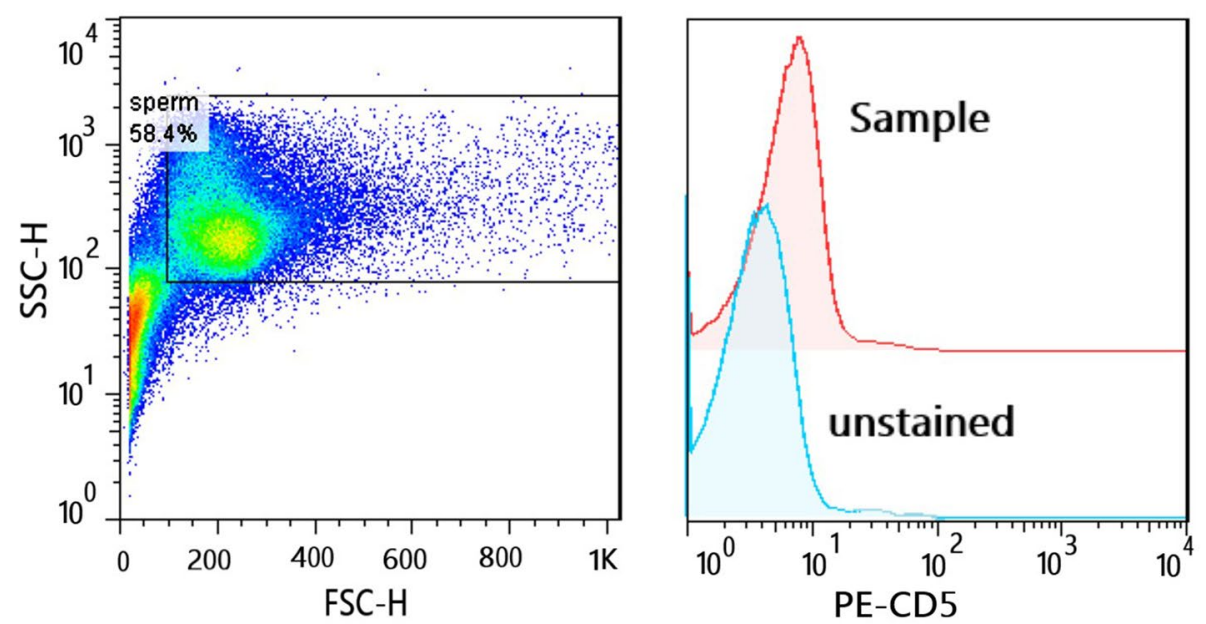

Fig. 1 Representative flow cytometry gating plots and histogram of unstained control and sample. Unstained control was used to differentiate the negative and positive population

trogocytosis process, can have important immune outcomes. As mentioned earlier, CD5 is generally known as an immunomodulatory molecule on $\mathrm{T}$ and $\mathrm{B}$ cells, and, like other immunoregulatory markers on lymphocytes such as PD1 and CTLA4, CD5 has the ability to modulate different signaling pathways in lymphocytes after activation through its extracellular domains [13]. Moreover, it is shown that some lymphocyte-interacting cells such as dendritic cells (DCs) express CD5 and this expression is related to their capacity of lymphocyte activation [12, 14]. Consequently, it is proposed that CD5 on the surface of DCs act through a homophilic interaction with CD5 on lymphocytes to regulate their functions [12]. Therefore, we suggest that CD5 in sperm plays the same role as it does in DCs. In the other words, spermatozoa, through presentation of $\mathrm{CD} 5$, may induce a regulatory phenotype on TCD4 and TCD8 cells and also B cells recruited to the female reproductive tract upon insemination, since, as stated previously, the semen-triggered immune reactions in the FRT need to be dominated by immunoregulatory mechanisms to culminate in normal pregnancy occurrence. Perhaps one of the reasons for hyper-immunity and excessive effector response in women suffering from recurrent spontaneous abortion (RSA) is due to disturbance in CD5 expression or signaling in the husband's spermatozoa.

\section{Conclusions}

This paper shows, for the first time, that spermatozoa express CD5 that is an immune regulator molecule. This result suggests that CD5 from spermatozoa may have some role in the induction of the regulatory immune response required for pregnancy occurrence.

\section{Abbreviations}

FRT: Female reproductive tract; TGF- $\beta$ : Transforming growth factor $\beta$; HLA: Human leukocyte antigen; CTLA-4: Cytotoxic T lymphocyte-associated protein 4; PD1: Programmed cell death protein 1.

\section{Acknowledgements}

The authors acknowledge the financial support of Isfahan University of Medical Sciences.

\section{Availability of data and materials:}

The datasets used and/or analyzed during the current study are available from the corresponding author on reasonable request.

\section{Authors' contributions}

N.S.: designed and performed experiments, analyzed data, and co-wrote the paper. M.T.: performed experiments and co-wrote the paper. R.A.: performed the experiments, co-wrote the paper, and supervised the research. D.W.: co-wrote, reviewed, and edited the paper. V.H.: performed the calculations. All authors have read and approved the manuscript.

\section{Funding}

Isfahan University of Medical Sciences. The funders had no role in study design, data collection and analysis, decision to publish, or preparation of the manuscript.

\section{Declarations}

\section{Ethics approval and consent to participate}

Written informed consent was obtained from all subjects who participated in this study. The protocol for this study was approved by the Ethics Committee of Isfahan University of Medical Sciences (Isfahan, Iran). The ethics committee approval letter number is IR.MUI.REC.1395.3.480.

\section{Consent for publication}

Not applicable.

\section{Competing interests}

The authors report no competing interests. 


\section{Author details}

${ }^{1}$ Asadabad School of Medical Sciences, Asadabad, Iran. ${ }^{2}$ Department of Immunology, School of Medicine, Isfahan University of Medical Sciences, Isfahan, Iran. ${ }^{3}$ Department of Immunology, Cellular and Molecular Research Center, Birjand University of Medical Sciences, Birjand, Iran. ${ }^{4}$ University of Aberdeen, Scotland, UK.

Received: 31 October 2020 Accepted: 10 January 2022

Published online: 18 January 2022

\section{References}

1. Moldenhauer LM et al (2009) Cross-presentation of male seminal fluid antigens elicits $T$ cell activation to initiate the female immune response to pregnancy. J Immunol 182(12):8080-8093

2. Robertson SA, Sharkey DJ (2001) The role of semen in induction of maternal immune tolerance to pregnancy. In: Seminars in immunology 13(4):243-254. Academic Press

3. Schuberth $\mathrm{H}$ et al (2008) Immunological responses to semen in the female genital tract. Theriogenology 70(8):1174-1181

4. Du Plessis SS, Gokul S, Agarwal A (2013) Semen hyperviscosity: causes, consequences, and cures. Front Biosci (Elite Ed) 5:224-231

5. Archana SS et al (2019) Immune regulatory molecules as modifiers of semen and fertility: a review. Mol Reprod Dev 86(11):1485-1504

6. Yao G-D et al (2014) Expression and potential roles of HLA-G in human spermatogenesis and early embryonic development. PLoS One 9(3):e92889

7. Avendano C et al (2009) Pregnancy-specific $\beta$-1-glycoprotein 1 and human leukocyte antigen-E mRNA in human sperm: differential expression in fertile and infertile men and evidence of a possible functional role during early development. Hum Reprod 24(2):270-277

8. Del Giudice PT et al (2010) Expression of the Fas-ligand gene in ejaculated sperm from adolescents with and without varicocele. J Assist Reprod Genet 27(2-3):103-109

9. Vick E, Mahadevan D (2016) Programming the immune checkpoint to treat hematologic malignancies. Expert Opin Investig Drugs 25(7):755-770

10. Voisinne G, Gonzalez de Peredo A, Roncagalli R (2018) CD5, an undercover regulator of TCR signaling. Front Immunol 9:2900

11. Saito $S$ et al (2010) Th1/Th2/Th17 and regulatory T-cell paradigm in pregnancy. Am J Reprod Immunol 63(6):601-610

12. Freitas CMT, Johnson DK, Weber KS (2018) T cell calcium signaling regulation by the co-receptor CD5. Int J Mol Sci 19(5)

13. Burgueño-Bucio E, Mier-Aguilar CA, Soldevila G (2019) The multiple faces of CD5. J Leukoc Biol 105(5):891-904

14. Li H et al (2019) CD5 on dendritic cells regulates CD4+ and CD8+ T cell activation and induction of immune responses. PLoS One 14(9):e0222301

15. Sereshki $\mathrm{N}$ et al (2019) Decreased toll-like receptor (TLR) 2 and 4 expression in spermatozoa in couples with unexplained recurrent spontaneous abortion (URSA). Iran J Allergy Asthma Immunol 18(6):701-706

16. Barraud-Lange $V$ et al (2007) Transfer of oocyte membrane fragments to fertilizing spermatozoa. FASEB J 21(13):3446-3449

\section{Publisher's Note}

Springer Nature remains neutral with regard to jurisdictional claims in published maps and institutional affiliations.

\section{Submit your manuscript to a SpringerOpen ${ }^{\circ}$ journal and benefit from:}

- Convenient online submission

- Rigorous peer review

- Open access: articles freely available online

- High visibility within the field

- Retaining the copyright to your article

Submit your next manuscript at $\boldsymbol{\nabla}$ springeropen.com 